\title{
The Role of Presystolic A Wave in the Differentiation of Non-obstructive Hypertrophic Cardiomyopathy and Athlete's Heart
}

\author{
Dilek KIRCI ${ }^{1}$, Muhammet Sayin ${ }^{2}$, and Gülay UZUN ${ }^{3}$ \\ ${ }^{1}$ Trabzon Kanuni Egitim ve Arastirma Hastanesi \\ ${ }^{2}$ Bulent Ecevit University \\ ${ }^{3}$ Trabzon Ahi Evren Thoracic and Cardiovascular Surgery Training and Research Hospital
}

May 18, 2020

\begin{abstract}
Background: Even though hypertrophic cardiomyopathy (HCM) can be distinguished from the athlete's heart with a difference in echocardiographic measurements, difficulties may be encountered in clinical practice from time to time. The aim of this study was to evaluate the use of presystolic A wave (PSAW) assessed by the Doppler echocardiography for differentiating between HCM and athlete's heart. Methods: A total of 52 subjects were included in the present study, of which, 27 had HCM and 25 were athletes. The pulsed Doppler assessment of the left ventricular outflow tract was performed on the ventricular face from the immediate proximal of the aortic valve on the apical five-chamber view. Allpatients were assessed for the presence of PSAW, and the velocity of this wave was recordedin PSAW positive subjects. Results: The frequency of PSAW was found to be higher in patients with HCM $[\mathrm{n}=12(44 \%)]$ than in athletes $[\mathrm{n}=4(16 \%)](\mathrm{p}=0.026)$. PSAW velocity measurements were observed to be higher in the HCM group; however, there was no statistical significance [53 ms (36-84)], [68 ms (35-193)], (p = 0.362). Conclusion: While there is a need for scaling-up similar studies, the current findings suggest that PSAW can be used as a distinguishing parameter for differentiating HCM and athlete's heart.
\end{abstract}

\section{Introduction}

Hypertrophic cardiomyopathy (HCM) has been defined as a condition with increased left ventricular wall thickness, which cannot be attributed to abnormal loading of the left ventricle alone. It is the most common hereditary cardiovascular disease, manifesting clinical, morphological, as well as genetic heterogeneity. ${ }^{(1)}$ HCM is the most important cause of sudden cardiac death in young athletes, and therefore its diagnosis is crucial.

The term athlete's heart is a clinical manifestation induced by a continuous and regular physical training program, and is characterized by two distinct and specific effects, viz, slow heart rate and enlarged heart. ${ }^{(2,3)}$ Adaptations of athlete's heart can mimic cardiovascular diseases and may often be confused with HCM.

Although the misdiagnosis of HCM may lead to inappropriate recommendations, including unnecessary treatment and exercising, failure to diagnose can lead to a risk of sudden death in athletes. HCM and athlete's heart can be distinguished from each other by various echocardiographic measurements. However, the differentiation may become difficult if there is no marked increase in the left ventricular wall thickness and the left ventricular outflow tract obstruction.

PSAW is a late diastolic activity, which can be frequently observed in the Doppler assessment of the left ventricular outflow tract. ${ }^{(4)}$ It is created by the reflection of the wave that is formed due to atrial contraction through the left ventricular wall. This reflection can take different forms in different clinical situations. 
The aim of this study was to evaluate the use of PSAW assessed by Doppler echocardiography, in the differentiation of HCM and athlete's heart.

\section{Methods}

\section{Patient Cohort}

Among the patients who applied to Cardiology Outpatient Clinics of the University of ... (Blinded) Thoracic and Cardiovascular Surgery Healthcare Application and Research Center between May 2017 and February 2019, and who were diagnosed with HCM in accordance with the diagnosis criteria of the European Cardiology Society [the criterion in the echocardiographic measurements is usually the maximum wall thickness [?]15 mm], the study included 27 patients who were not diagnosed with the following:other causes that can lead to left ventricular hypertrophy (e.g., infiltrative diseases such as amyloidosis, chronic kidney failure, aortic stenosis, uncontrolled hypertension), secondary hypertension, severe renal and hepatic dysfunction, left ventricular systolic dysfunction, severe primary valve disease, mitral annulus calcification, HCM-related septal ablation or a past surgical therapy, presence of AF in ECG monitoring, severe coronary artery disease (presence of $>50 \%$ blockage in at least one of the coronary arteries), history of myocardial infarction, other congenital cardiovascular disease, atrial fibrillation, any grade of atrioventricular block, severe bradycardia, sick sinus syndrome, presence of cardiac pacemaker, presence of complete branch block, electrolyte abnormality, malignancy, pregnancy, asthma or advanced chronic obstructive pulmonary disease, aortic diseases and connective tissue disorders.

The study also included 25 athletes who have played sports professionally between the same dates in different sport branches for at least five years and who were not known to exhibit any cardiovascular disease or cardiologic symptoms. ${ }^{(5)}$

Approval for the study was obtained from the ethics committee of Trabzon Kanuni Training and Research Hospital. Verbal and written informed consents were obtained from the subjects included in the study.

\section{General Assessment and Blood Pressure Measurements}

The medical history of all the patients wascollected and their physical examination was performed. Body mass index and body surface area of all the patients were calculated by measuring their height and weight. Systolic and diastolic blood pressures were measured, and electrocardiography was taken. Cardiovascular disease risk factors were also recorded.

\section{Echocardiography}

Transthoracic echocardiography was performed on the patients after allowing them to rest in the echocardiography room for $15 \mathrm{~min}$ in a lying position. The echocardiographic assessment was performed with the VingMedSystemFiveinstrument with $2.5 \mathrm{MHz}$ probe (GE MedicalSystem, Hortan, Norway) in the left lateral position, using standard methods while the patient was breathing quietly. Electrocardiographic follow-up was performed simultaneously with the electrocardiography of the echocardiography device throughout the assessment. In the initial echocardiographic assessment, the presence of any pathology was eliminated using standard methods with two-dimensional color Doppler and intermittent flow tissue Doppler on the parasternal long axis, short axis, apical 4 and 2 chamber views, respectively.

Pulsed Doppler assessment of the left ventricular outflow tract was performed on the apical 5 chamber view on the ventricular face immediately proximal to the aortic valve. All patients were evaluated for the presence of PSAW, andthe PSAW velocities were recorded for subjects in which these were detected.

Additionally, echocardiographic parameters such as left atrial diameter, left ventricular end-systolic diameter, left ventricular end-diastolic diameter, septal and posterior wall thickness, early diastolic mitral flow rate (E) and late diastolic flow rate (A) were measured in all patients.

\section{Statistical analysis}


"SPSS Windows Version 19" (SPSS Inc. Chicago, Illinois, USA) software package was used for statistical analyses of the findings obtained in our study. Values for continuous variables were given as median (minimum-maximum); values for categorical variables were given as numbers and percentages. "Chi-Square Test" was used in the evaluation of categorical variables. "Mann-Whitney U Test" was used forthe evaluation of continuous variables. The difference was considered statistically significant if the p-value calculated for all statistical analyseswasless than 0.05 .

\section{Results}

The clinical and demographic characteristics of the patients arelisted in Table 1. A total of 52 cases were included in the current study, out of which 27 were patients with HCM and 25 were athletes. Age was significantly higher in the HCM group (50 (20-75), 26 (18-59), p <0.001).

Echocardiographic parameters are shown in Table 2. PSAW was observed to be higher in the group with HCM than in the control group (Figure 1). There were no significant differences between the PSAW velocities.

\section{Discussion}

In this study, PSAW was found to be more commonly observed in HCM patients compared to athletes. PSAW velocity values were observed to be higher in the HCM group; however, the increase was not statistically significant.

PSAW is a late diastolic wave that can be frequently observed in the Doppler assessment of the left ventricular outflow tract. ${ }^{(4)}$ However, its frequency, clinical significance and relationship with other echocardiographic parameters have not been fully established. There are different reportsindicating whether it is a normal phenomenon or a marker of pathological conditions. The formation mechanism of diastolic waves in the left ventricular outflow tract was demonstrated by Panaviotou and Byrd in 1990. ${ }^{(6)}$ This study suggested that diastolic waves in the left ventricular outflow tract are a normal phenomenon and the responsible mechanism originates from fluid mechanics, defined as "ring vortex". According to this mechanism, the diastolic filling flow coming from the left atrium to the left ventricle passes through the mitral valve and is directed to the left ventricular outflow tract, both by the vortex effect and through reflectionfrom the apex. This results in the appearance of diastolic flow waves with Doppler in the left ventricular outflow tract.

Left ventricular wall thicknesses were observed to benaturally higher in patients with HCM. In the abovecitedstudy, conducted by Panayiotou and Byrd, PSAW was observed more frequently in individuals with left ventricular hypertrophy, in comparison to those without it. ${ }^{(6)}$ Similar to the findings of the cited study, our study also detected a higher frequency of PSAW in patients with HCM. In their study, Panayiotou and Byrd detected higher PSAW velocities in individuals with left ventricular hypertrophy than those without. In our study too, PSAW velocities were measured higher in the HCM group, even though the values were not statistically significant.

Although PSAW is not expected to occur in the left ventricular outflow tract under normal conditions, PSAW can be encountered in different patient groups, with diastolic dysfunction. In a previous study conducted by Mittal et al., PSAW has been shown to be associated with left ventricular diastolic dysfunction. ${ }^{(7)}$ In our study too, HCM patients had deterioration in their left ventricular diastolic functions, and this may have caused PSAW to be observed more frequently in patients with HCM. It is known that left ventricular diastolic dysfunction develops with aging. ${ }^{(8)}$ Our patient cohort was older than our control group, and this may have contributed to more frequent observation of left ventricular diastolic dysfunction, and thus PSAW in patients with HCM. However, the most frequently observed pathophysiological event in HCM is known to be a diastolic dysfunction. ${ }^{(9)}$ It is independent of the grade and location of the left ventricular hypertrophy and may appear with or without pressure gradient or symptom. ${ }^{(10)}$ Therefore, we believe that the presence of age differences between the groups does not constitute a limitation.

Finocchiaro et al. evaluated Doppler parameters in HCM patients. ${ }^{(11)}$ In this study, Mitral E wave velocity was found to be lower in HCM patients. Similarly, in our study, Mitral E wave velocity was found lower in HCM patients but was not statistically significant. Finocchiaro et al. detected higher Mitral A wave velocity 
in HCM patients. Similarly, in our study, Mitral A wave velocity was found to be higher in patients with HCM. PSAW is the reflection of the mitral A wave. The more frequent occurrence of PSAW in the HCM group may also be originating from larger mitral A wave velocity.

Finocchiaro et al. also evaluated tissue Doppler parameters, ${ }^{(11)}$ wherein they detected tissue Doppler velocities to be lower in patients with HCM. In our study, tissue Doppler values obtained from both the septal and lateral wall were found to be lower in the HCM group compared to the athletes. Finocchiaro et al. detected the E/E' value to be higher in the HCM group,and similar values were observed in our study.

\section{Limitations}

The most significant limitation was that the number of patients included in the study was insufficient. Since the patients with HCM who applied to our hospital have been included in the study, they may not be generalized to the general population. Coronary artery patients were excluded from the study, but no invasive test was performed to rule out coronary artery disease. There was a difference inthe age between the HCM and the athlete groups.

\section{Conclusion}

In our study, the frequency and velocity of PSAW were higher in the HCM group compared to the athlete group. The current findings indicated that more frequent PSAW was caused by higher left ventricular wall thickness and stiffness, accompanied by left ventricular diastolic dysfunction, and higher mitral A wave velocity in HCM patients. Although there is a need for scaling-up such studies, PSAW can be used as a distinguishing parameter in the differentiation of HCM and athlete's heart, along with other accompanying findings.

\section{Acknowledgments}

None.

\section{Funding Sources}

None.

\section{Disclosures}

The authors have no conflicts of interest to disclose.

\section{Declarations of interest}

None.

\section{References}

1. Maron BJ. Hypertrophic cardiomyopathy: A systematic review. JAMA 2002;287:1308-20.

2. Pelliccia A, Culasso F, Di Paolo FM, Maron BJ. Physiologic left ventricular cavity dilatation in elite athletes. Ann Intern Med 1999;130:23-31.

3. Pelliccia A, Maron BJ, Spataro A, Proschan MA, Spirito P. The upper limit of physiologic cardiac hypertrophy in highly trained elite athletes. N Engl J Med 1991;32:295-301.

4. Armstrong WF, Ryan T, Feigenbaum H. Feigenbaum's Echocardiography. Philadelphia: Wolters Kluwer Health/Lippincott Williams \&Wilkins, 2010:545.

5. Elliott PM, Anastasakis A, Borger MA, Borggrefe M, Cecchi F, Charron P, et al. 2014 ESC Guidelines on diagnosis and management of hypertrophic cardiomyopathy: The Task Force for the Diagnosis and Management of Hypertrophic Cardiomyopathy of the European Society of Cardiology (ESC). Eur Heart J 2014;35:2733-79. 
6. Panayiotou H, Byrd BF 3rd. Origin and significance of diastolic Doppler flow signals in the left ventricular out flow tract. J Am CollCardiol 1990;16:1625-1631.

7. Mittal SR, Pancholi N. Left ventricular out flow tract presystolic flow velocity -another marker of left ventricular diastolic function.Int J Cardiovasc Imaging 2002;18:249-256.

8. Cheng JW, Nayar M. A review of heart failure management in the elderly population. Am J GeriatrPharmacother 2009; 7(5):233-249. 9. Nagueh SF, Lakkis NM, Middleton KJ, et al. Doppler estimation of left ventricular filling pressures in Patients with hypertrophic cardiomyopathy. Circulation 1999;99:254-61.

10. Nagueh SF, Bachinski LL, Meyer D, et al. Tissue Doppler imaging consistently detects myocardial abnormalities in patients with hypertrophic cardiomyopathy and provides a novel means for an early diagnosis before and independently of hypertrophy. Circulation 2001;104(2):128-30.

11. Finocchiaro G, Dhutia H, D'Silva A, et al. Role of Doppler Diastolic Parameters in Differentiating Physiological Left Ventricular Hypertrophy from Hypertrophic Cardiomyopathy J Am SocEchocardiogr 2018;31(5):606-613. 